\title{
Runge-Kutta Methods with Minimum Error Bounds
}

\author{
By Anthony Ralston
}

1. Introduction. Numerical methods for the solution of ordinary differential equations may be put in two categories-numerical integration (e.g., predictorcorrector) methods and Runge-Kutta methods. The advantages of the latter are that they are self-starting and easy to program for digital computers but neither of these reasons is very compelling when library subroutines can be written to handle systems of ordinary differential equations. Thus, the greater accuracy and the error-estimating ability of predictor-corrector methods make them desirable for systems of any complexity. However, when predictor-corrector methods are used, Runge-Kutta methods still find application in starting the computation and in changing the interval of integration.

If, then, Runge-Kutta methods are considered in the context of using them for starting and for changing the interval, matters such as stability [2], [3] and minimization of roundoff errors [4] are not significant. Also, simplifying the coefficients so that the computation will be speeded up is not important and, on modern computers, minimization of storage [4] is seldom important. In fact, the only criterion of significance in judging Runge-Kutta methods in this context is minimization of truncation error. It is the purpose of this paper to derive Runge-Kutta methods of second, third and fourth order which have minimum truncation error bounds of a specified type. We will consider only the case of integrating a single first-order differential equation because this is the only tractable case analytically. But it seems reasonable to assume that methods which are best in a truncation error sense for one equation will be at least nearly best for systems of equations.

2. The General Equations. For the solution of the equation

$$
y^{\prime}=f(x, y) \quad y\left(x_{0}\right)=y_{0}
$$

at a sequence of points $x_{1}, x_{2}, \cdots$ the general Runge-Kutta method of order $m$ is

$$
y_{n+1}-y_{n} \equiv k=\sum_{i=1}^{m} w_{i} k_{i}
$$

where $y_{r}=y\left(x_{r}\right)$, the $w_{i}$ 's are constants and

$$
k_{i}=h_{n} f\left(x_{n}+\alpha_{i} h_{n}, y_{n}+\sum_{j=1}^{i-1} \beta_{i j} k_{j}\right)
$$

with $h_{n}=x_{n+1}-x_{n}$ and $\alpha_{1}=0$. By choosing the $\alpha_{i}$ 's and $\beta_{i j}$ 's properly the expansion of the right hand side of equation (2.2) about $\left(x_{n}, y_{n}\right)$ in powers of $h_{n}$ can be made identical with the Taylor series expansion of $k$ about $x_{n}$ through the term

Received September 22, 1961. 
in $h_{n}{ }^{m}$. One set of constraints on the $\alpha_{i}$ 's and $\beta_{i j}$ 's that always results is

$$
\alpha_{i}=\sum_{j=1}^{i-1} \beta_{i j}
$$

The error to be added to the right hand side of equation (2.2) to make it an exact relationship consists of a term $E h_{n}^{m+1}$, where $E$ depends on $f(x, y)$, plus terms of higher degree in $h_{n}$. For the classical fourth-order method of Kutta [6] a bound on $E$ has been found by Lotkin [7] who improved on a bound of Bieberbach [1]. We will derive bounds on $E$ for Runge-Kutta methods of second, third and fourth order in the same form as Lotkin:

$$
|E|<c M L^{m}
$$

where $c$ is a constant and, in a region $R$ about $\left(x_{n}, y_{n}\right)^{*}$

$$
|f(x, y)|<M \text { and } \frac{\partial^{i+j} f}{\partial x^{i} \partial y^{j}}<L^{i+j} / M^{j-1}
$$

where $M$ and $L$ are constants and $i+j \leqq m$.

3. Second-Order Systems. The coefficients to be determined are $w_{1}, w_{2}$, $\alpha_{2}$ and $\beta_{21}$. Matching powers of $h_{n}$ through $h_{n}{ }^{2}$ imposes three constraints (see, for example, [5]) which leads to the following one-parameter family:

$$
w_{1}=1-1 /\left(2 \alpha_{2}\right) \quad w_{2}=1 /\left(2 \alpha_{2}\right)
$$

with $\beta_{21}=\alpha_{2}$ following from equation (2.4). The coefficient $c_{2}$ of $h_{n}{ }^{3}$ is given by

$$
c_{2}=\left[\left(\frac{1}{6}\right)-\left(\alpha_{2}^{2} w_{2} / 2\right)\right] D^{2} f+\left(\frac{1}{6}\right) f_{y} D f
$$

where

$$
D=\partial / \partial x+f_{n}(\partial / \partial y) \quad f_{n}=f\left(x_{n}, y_{n}\right) .
$$

Using (3.1) and (3.3) in (3.2) and the notation of (2.6), a bound on $E$ is given by

$$
|E|<\left[4\left|\left(\frac{1}{6}\right)-\left(\alpha_{2} / 4\right)\right|+\frac{1}{3}\right] M L^{2} \text {. }
$$

Clearly the minimum bound will be achieved if we set $\alpha_{2}=\frac{2}{3}$ which leads to the well known formula [5]

$$
y_{n+1}-y_{n}=\frac{1}{4} h_{n} f\left(x_{n}, y_{n}\right)+\left(\frac{3}{4}\right) h_{n} f\left(x_{n}+\left(\frac{2}{3}\right) h_{n}, y_{n}+\left(\frac{2}{3}\right) h_{n} f_{n}\right)
$$

for which the right-hand side of (3.4) becomes $\left(\frac{1}{3}\right) M L^{2}$.

4. Third-Order Systems. In this case the coefficients form a two-parameter family given by

$$
\begin{aligned}
& w_{1}=1+\left[2-3\left(\alpha_{2}+\alpha_{3}\right)\right] / 6 \alpha_{2} \alpha_{3} \\
& w_{2}=\left(3 \alpha_{3}-2\right) /\left[6 \alpha_{2}\left(\alpha_{3}-\alpha_{2}\right)\right] \\
& w_{3}=\left(2-3 \alpha_{2}\right) /\left[6 \alpha_{3}\left(\alpha_{3}-\alpha_{2}\right)\right] \\
& \beta_{32}=\left[\alpha_{3}\left(\alpha_{3}-\alpha_{2}\right)\right] /\left[\alpha_{2}\left(2-3 \alpha_{2}\right)\right]
\end{aligned}
$$

* This region must, of course, include all values of $x$ and $y$ in equations (2.2) and (2.3). 
when $\alpha_{2} \neq 0, \alpha_{3} \neq 0$, and $\alpha_{2} \neq \alpha_{3}$. When $\alpha_{3}=0$ there is a one-parameter family given by

$$
\begin{array}{ll}
\alpha_{2}=\frac{2}{3} & w_{1}=\frac{1}{4}-w_{3} \\
\beta_{32}=1 /\left(4 w_{3}\right) & w_{2}=\frac{3}{4} .
\end{array}
$$

When $\alpha_{2}=\alpha_{3}=\frac{2}{3}$ there is a one-parameter family given by

$$
w_{1}=\frac{1}{4} \quad w_{2}=\frac{3}{4}-w_{3} \quad \beta_{32}=1 /\left(4 w_{3}\right)
$$

No solutions other than (4.1), (4.2), and (4.3) are possible.

The coefficient $c_{3}$ of $h_{n}{ }^{4}$ is given by

$$
\begin{aligned}
c_{3} & =\left[(1 / 4 !)-(1 / 3 !)\left(\alpha_{2}{ }^{2} w_{2}+\alpha_{3}{ }^{2} w_{3}\right)\right] D^{3} f \\
& +\left[(1 / 4 !)-(1 / 2 !) \alpha_{2}{ }^{2} \beta_{32} w_{3}\right] f_{y} D^{2} f \\
& +\left[(3 / 4 !)-\alpha_{2} \alpha_{3} \beta_{32} w_{3}\right) D f D f_{y}+(1 / 4 !) f_{y}{ }^{2} D f .
\end{aligned}
$$

Substituting (3.3) and (4.1) into (4.4), combining terms, taking absolute values, and using (2.6) leads to the following bound on $E$ :

$$
|E|<\left[8\left|a_{1}\right|+\left|a_{2}\right|+\left|2 a_{2}+a_{3}\right|+\left|a_{2}+a_{3}\right|+2\left|a_{3}\right|+2\left|a_{4}\right|\right] M L^{3}
$$

where

$$
\begin{aligned}
& a_{1}=\left(\frac{1}{24}\right)-\left(\frac{1}{36}\right)\left[2\left(\alpha_{2}+\alpha_{3}\right)-3 \alpha_{2} \alpha_{3}\right] \\
& a_{2}=\left(\frac{1}{24}\right)-\alpha_{2} / 12 \\
& a_{3}=\left(\frac{1}{8}\right)-\alpha_{3} / 6 \quad a_{4}=\frac{1}{24} .
\end{aligned}
$$

A simple analysis shows that the coefficient of $M L^{3}$ in (4.5) will be minimized if $\alpha_{2}=\frac{1}{2}$ and $\alpha_{3}=\frac{3}{4}$ in which case we get

$$
|E|<\left(\frac{1}{9}\right) M L^{3} \text {. }
$$

If (4.2) instead of (4.1) is used in (4.4) the bound on $E$ is

$$
|E|<\left(\frac{2}{3}\right) M L^{3}
$$

independent of the value of $w_{3}$. If (4.3) is used the bound, again independent of $w_{3}$, is

$$
|E|<\left(\frac{1}{4}\right) M L^{3}
$$

Thus, for third-order Runge-Kutta methods the minimum error bound of the type we are considering is given by (4.7). In this case equations (2.2) and (2.3) become

$$
y_{n+1}-y_{n}=\left(\frac{2}{9}\right) k_{1}+\left(\frac{1}{3}\right) k_{2}+\left(\frac{4}{9}\right) k_{3}
$$

where

$$
\begin{aligned}
& k_{1}=h_{n} f\left(x_{n}, y_{n}\right) \\
& k_{2}=h_{n} f\left(x_{n}+\frac{1}{2} h_{n}, y_{n}+\frac{1}{2} k_{1}\right) \\
& k_{3}=h_{h} f\left(x_{n}+\left(\frac{3}{4}\right) h_{n}, y_{n}+\left(\frac{3}{4}\right) k_{2}\right) .
\end{aligned}
$$


5. Fourth-Order Systems. Again, the coefficients form a two-parameter family. They are given by

$$
\begin{aligned}
w_{1} & =\frac{1}{2}+\left[1-2\left(\alpha_{2}+\alpha_{3}\right)\right] / 12 \alpha_{2} \alpha_{3} \\
w_{2} & =\left(2 \alpha_{3}-1\right) /\left[12 \alpha_{2}\left(\alpha_{3}-\alpha_{2}\right)\left(1-\alpha_{2}\right)\right] \\
w_{3} & =\left(1-2 \alpha_{2}\right) /\left[12 \alpha_{3}\left(\alpha_{3}-\alpha_{2}\right)\left(1-\alpha_{3}\right)\right] \\
w_{4} & =\frac{1}{2}+\left[2\left(\alpha_{2}+\alpha_{3}\right)-3\right] /\left[12\left(1-\alpha_{2}\right)\left(1-\alpha_{3}\right)\right] \\
\alpha_{4} & =1 \quad \beta_{32}=\left[\alpha_{3}\left(\alpha_{3}-\alpha_{2}\right)\right] /\left[2 \alpha_{2}\left(1-2 \alpha_{2}\right)\right] \\
\beta_{42} & =\frac{\left(1-\alpha_{2}\right)\left[\alpha_{2}+\alpha_{3}-1-\left(2 \alpha_{3}-1\right)^{2}\right]}{2 \alpha_{2}\left(\alpha_{3}-\alpha_{2}\right)\left[6 \alpha_{2} \alpha_{3}-4\left(\alpha_{2}+\alpha_{3}\right)+3\right]} \\
\beta_{43} & =\frac{\left(1-2 \alpha_{2}\right)\left(1-\alpha_{2}\right)\left(1-\alpha_{3}\right)}{\alpha_{3}\left(\alpha_{3}-\alpha_{2}\right)\left[6 \alpha_{2} \alpha_{3}-4\left(\alpha_{2}+\alpha_{3}\right)+3\right]}
\end{aligned}
$$

when $\alpha_{2} \neq 0, \alpha_{3} \neq 0, \alpha_{2} \neq 1, \alpha_{3} \neq 1$, and $\alpha_{2} \neq \alpha_{3}$. The other possible solutions are

and

$$
\begin{array}{lll}
\alpha_{2}=\alpha_{3}=\frac{1}{2} \quad \alpha_{4}=1 \\
w_{1}=\frac{1}{6} & w_{2}=\left(\frac{2}{3}\right)-w_{3} & w_{4}=\frac{1}{6} \\
\beta_{32}=1 /\left(6 w_{3}\right) & \beta_{42}=1-3 w_{3} & \beta_{43}=3 w_{3}
\end{array}
$$

and

$$
\begin{array}{lll}
\alpha_{2}=1 & \alpha_{3}=\frac{1}{2} & \alpha_{4}=1 \\
w_{1}=\frac{1}{6} & w_{2}=\frac{1}{6}-w_{4} & w_{3}=\frac{2}{3} \\
\beta_{32}=\frac{1}{8} & \beta_{42}=-1 /\left(12 w_{4}\right) & \beta_{43}=1 /\left(3 w_{4}\right)
\end{array}
$$

$$
\begin{array}{lll}
\alpha_{2}=\frac{1}{2} & \alpha_{3}=0 & \alpha_{4}=1 \\
w_{1}=\left(\frac{1}{6}\right)-w_{3} & w_{2}=\frac{2}{3} & w_{4}=\frac{1}{6} \\
\beta_{32}=1 /\left(12 w_{3}\right) & \beta_{42}=\frac{3}{2} & \beta_{43}=6 w_{3} .
\end{array}
$$

For $\alpha_{2}=0$ and $\alpha_{3}=1$ no solutions are possible. Proceeding as before, a tedious computation leads to a bound on the error of

$$
\begin{aligned}
|E|<\left[16\left|b_{1}\right|+4\left|b_{2}\right|+\mid b_{2}\right. & +3 b_{3}|+| 2 b_{2}+3 b_{3}|+| b_{2}+b_{3} \mid \\
+\left|b_{3}\right|+8\left|b_{4}\right|+\left|b_{5}\right| & +\left|2 b_{5}+b_{7}\right|+\left|b_{5}+b_{6}+b_{7}\right| \\
& \left.+\left|b_{6}\right|+\left|2 b_{6}+b_{7}\right|+\left|b_{7}\right|+2\left|b_{8}\right|\right] M L^{4}
\end{aligned}
$$

where

$$
\begin{aligned}
& b_{1}=\left(\frac{1}{12 \sigma}\right)-\left(\frac{1}{24}\right)\left(\alpha_{2}{ }^{4} w_{2}+\alpha_{3}{ }^{4} w_{3}+w_{4}\right) \\
& b_{2}=\left(\frac{1}{20}\right)-\frac{1}{2}\left[\alpha_{2} \alpha_{3}^{2} \beta_{32} w_{3}+\left(\alpha_{2} \beta_{42}+\alpha_{3} \beta_{43}\right) w_{4}\right] \\
& b_{3}=\left(\frac{1}{12 \sigma}\right)-\left(\frac{1}{6}\right)\left[\alpha_{2}^{3} \beta_{32} w_{3}+\left(\alpha_{2}^{3} \beta_{42}+\alpha_{3}^{3} \beta_{43}\right) w_{4}\right] \\
& b_{4}=\left(\frac{1}{30}\right)-\frac{1}{2}\left[\alpha_{2}^{2} \alpha_{3} \beta_{32} w_{3}+\left(\alpha_{2}^{2} \beta_{42}+\alpha_{3}^{2} \beta_{43}\right) w_{4}\right] \\
& b_{5}=\left(\frac{1}{12} \sigma\right)-\frac{1}{2} \alpha_{2}^{2} \beta_{32} \beta_{43} w_{4} \\
& b_{6}=\left(\frac{1}{4 \sigma}\right)-\frac{1}{2}\left[\alpha_{2}{ }^{2} \beta_{32}{ }^{2} w_{3}+\left(\alpha_{2} \beta_{42}+\alpha_{3} \beta_{43}\right)^{2} w_{4}\right] \\
& b_{7}=\left(\frac{7}{12 \delta}\right)-\alpha_{2}\left(1+\alpha_{3}\right) \beta_{32} \beta_{43} w_{4} \quad b_{8}=\frac{1}{12} \overline{0} \text {. }
\end{aligned}
$$


Finding those values of $\alpha_{2}$ and $\alpha_{3}$ which, after substitution of (5.1) into (5.6), minimize the coefficient of $M L^{4}$ in (5.5) is extremely tedious but not impossible. The result is $\alpha_{2}=.4$ and $\alpha_{3}=\left(\frac{7}{8}\right)-\left(\frac{3}{16}\right)(5)^{1 / 2}$ and in this case (5.5) becomes

$$
|E|<5.46 \times 10^{-2} M L^{4} \text {. }
$$

If (5.2) is used instead of $(5.1)$, the bound is minimized if $w_{3}=\frac{5}{3}$, in which case

$$
|E|<\left(\frac{13}{180}\right) M L^{4}=7.22 \times 10^{-2} M L^{4} \text {. }
$$

Using (5.3) the bound is minimized if $w_{4}=\frac{1}{5} \frac{0}{1}$, in which case

$$
|E|<\left(\frac{71}{360}\right) M L^{4}=19.72 \times 10^{-2} M L^{4} \text {. }
$$

Using (5.4) the bound is minimized when $w_{3}=-\frac{5}{78}$ in which case

$$
|E|<\left(\frac{127}{72}\right) M L^{4}=17.64 \times 10^{-2} M L^{4} \text {. }
$$

Thus, the best bound is given by (5.7) and the complete set of equations in this case (correct to eight decimal places) is

$$
y_{n+1}-y_{n}=.17476028 k_{1}-.55148066 k_{2}+1.20553560 k_{3}+.17118478 k_{4}
$$

where

$$
\begin{aligned}
& k_{1}=h_{n} f\left(x_{n}, y_{n}\right) \\
& k_{2}=h_{n} f\left(x_{n}+.4 h_{n}, y_{n}+.4 k_{1}\right) \\
& k_{3}=h_{n} f\left(x_{n}+.45573725 h_{n}, y_{n}+.29697761 k_{1}+.15875964 k_{2}\right) \\
& k_{4}=h_{n} f\left(x_{n}+h_{n}, y_{n}+.21810040 k_{1}-3.05096516 k_{2}+3.83286476 k_{3}\right) .
\end{aligned}
$$

The coefficients for the classical fourth-order method of Kutta are given by (5.2) with $w_{3}=\frac{1}{3}$. For this case Lotkin [7] found the bound

$$
|E|<\left(\frac{73}{720}\right) M L^{4}=10.14 \times 10^{-2} M L^{4}
$$

which has a coefficient almost twice as great as that given by (5.7). This classical method may be considered a special case of the class of methods in which $\alpha_{3}=1-$ $\alpha_{2}$. The method of this class for $\alpha_{2} \neq \frac{1}{2}$ which has the minimum error bound also has reasonably simple coefficients and, therefore, is of some interest since it provides an improvement of the classical method even for hand computation. This method also has $\alpha_{2}=.4$ and the complete set of equations is

$$
y_{n+1}-y_{n}=\left(\frac{1}{72}\right)\left(11 k_{1}+25 k_{2}+25 k_{3}+11 k_{4}\right)
$$

where

$$
\begin{aligned}
& k_{1}=h_{n} f\left(x_{n}, y_{n}\right) \\
& k_{2}=h_{n} f\left(x_{n}+\left(\frac{2}{5}\right) h_{n}, y_{n}+\left(\frac{2}{5}\right) k_{1}\right) \\
& k_{3}=h_{n} f\left(x_{n}+\left(\frac{3}{5}\right) h_{n}, y_{n}-\left(\frac{3}{20}\right) k_{1}+\left(\frac{3}{4}\right) k_{2}\right) \\
& k_{4}=h_{n} f\left(x_{n}+h_{n}, y_{n}+\left(\frac{1}{44}\right)\left(19 k_{1}-15 k_{2}+40 k_{3}\right)\right) .
\end{aligned}
$$

The error bound is given by

$$
|E|<\left(\frac{127}{1650}\right) M L^{4}=7.70 \times 10^{-2} M L^{4} .
$$


For completeness we list here the error bounds for two other fourth-order RungeKutta procedures which have appeared prominently in the literature:

(1) $\alpha_{2}=\frac{1}{3}, \alpha_{3}=\frac{2}{3}$ due to Kutta [6].

$$
|E|<\left(\frac{107}{1080}\right) M L^{4}=9.91 \times 10^{-2} M L^{4}
$$

(2) $\alpha_{2}=\frac{1}{2}, \alpha_{3}=\frac{1}{2}, w_{3}=1+1 /(2)^{1 / 2}$ due to Gill [4].

$$
|E|<\left[\left(\frac{53}{360}\right)-\left(\frac{1}{12}\right)\left(\frac{1}{2}^{\frac{1}{2}}\right)\right] M L^{4}=8.83 \times 10^{-2} M L^{4} \text {. }
$$

6. Numerical Examples and Conclusions. It is standard procedure in papers of this kind to finish with some numerical examples that illustrate how favorably the methods derived compare with other methods. Milne [8] remarks that especially in papers dealing with Runge-Kutta methods examples tend to be chosen which favorably illustrate the derived method. In fact, it is difficult to choose meaningful examples to illustrate Runge-Kutta methods and the reason for this is clear; the complicated nature of the error term makes it difficult to choose functions $f(x, y)$

\begin{tabular}{|c|c|c|c|}
\hline Step Size $(h)$ & Number of Steps & Method & $\begin{array}{l}\text { Magnitude of Error } \\
\text { after Number of Steps } \\
\text { in Column } 2\left(\times 10^{8}\right)\end{array}$ \\
\hline .1 & $\overline{5}$ & $\begin{array}{l}\mathrm{A} \\
\mathrm{B} \\
\mathrm{C}\end{array}$ & $\begin{array}{l}12 \\
34 \\
65\end{array}$ \\
\hline .1 & 10 & $\begin{array}{l}\mathrm{A} \\
\mathrm{B} \\
\mathrm{C}\end{array}$ & $\begin{array}{r}75 \\
138 \\
152\end{array}$ \\
\hline .2 & 5 & $\begin{array}{l}\mathrm{A} \\
\mathrm{B} \\
\mathrm{C}\end{array}$ & $\begin{array}{l}1190 \\
2061 \\
2492\end{array}$ \\
\hline
\end{tabular}

TABLE 1

TABLE 2

Errors in Integration of $y^{\prime}=\left[e^{x}\left(y^{3}+x y^{3}+1\right)\right] /\left[3 y^{2}\left(x e^{x}-6\right)\right]$, $y(0)=1$ (Solution: $y=\left[\left(e^{x}+5\right) /\left(6-x e^{x}\right)\right]^{1 / 3}$

\begin{tabular}{c|c|c|c}
\hline Step Size $(h)$ & Number of Steps & Method & $\begin{array}{c}\text { Magnitude of Error } \\
\text { after Number of Steps } \\
\text { in Column 2 }\left(\times 10^{7}\right)\end{array}$ \\
\hline .1 & 5 & A & 5 \\
& B & 10 \\
& 10 & C & 8 \\
& A & 6 \\
\hline
\end{tabular}


which really serve as a test and at the same time are such that the differential equation (2.1) can be solved analytically.

Here, for the sake of illustration and to indicate that the derivations in this paper were performed correctly, we present in Tables 1 and 2 the results of two simple computations comparing three Runge-Kutta methods:*

Method A-Equations (5.11) and (5.12)

Method B-Equations (5.14) and (5.15)

Method C-The classical method with coefficients given by (5.2) with $w_{3}=\frac{1}{3}$. In the example in Table 1 method A compares quite favorably with the other two while the comparison in Table 2 is not nearly so favorable. The error bound for method $\mathrm{A}$ for the example in Table 1 calculated from (5.7) varies from about $10^{-5}$ to $10^{-6}$ as $x$ goes from 0 to .5, which is indicative of the fact that error bounds of the type we have derived here are generally quite conservative. We note that it is only a matter of a little ingenuity to find other examples to make method A appear more or less favorable in comparison with other Runge-Kutta methods.

In conclusion, we emphasize again the main point of this paper which is likely to be obscured by giving examples. This is that, if Runge-Kutta methods are to be used to start the solution and to change the interval, one is interested only in being able to bound the truncation error as well as possible. Thus, that method which allows the smallest bound to be put on the error is in this sense best. Therefore, we conclude that on a digital computer equations (5.11) and (5.12) should be used when a fourth-order Runge-Kutta method is to be used for starting the solution or changing the interval. Similarly, equations (4.10) and (4.11) are recommended as a third-order system and equation (3.5) as a second-order system.

Stevens Institute of Technology

Hoboken, New Jersey 1944.

1. L. Bieberbach, Theorie der Differentialgleichungen, Dover Publications, New York,

2. J. W. CARR III, “Error bounds for the Runge-Kutta single-step integration process," J. Assoc. Comput. Mach., v. 5, 1958, p. 39-44.

3. B. A. Galler \& D. P. Rozenberg, "A generalization of a theorem of Carr on error bounds for Runge-Kutta procedures," J. Assoc. Comput. Mach., v. 7, 1960, p. 57-60.

4. S. GiLl, "A process for step-by-step integration of a differential equation in an automatic digital computing machine," Proc. Cambridge Philos. Soc., v. 47, 1951, p. 96-108.

5. Z. Kopal, Numerical Analysis, John Wiley \& Sons, New York, 1955.

6. W. KUTTA, "Beitrag zur näherungsweiser Integration totaler Differentialgleichungen," Z. Math. Phys., v. 46, 1901, p. 435-453.

7. M. LotKin, "On the accuracy of Runge-Kutta's method," MTAC, v. 5, 1951, p. 128-

8. W. E. Milne, "Note on the Runge-Kutta method," J. Res. Nat. Bur. Standards, v. 44, 1950, p. 549-550.

* These calculations as well as some calculations which located roughly the values of $\alpha_{2}$ and $\alpha_{3}$ in (5.12) were performed on the IBM 1620 computer at the Stevens Institute of Technology. This computer is partially supported by the National Science Foundation. 